\title{
Regular approximation of singular Sturm-Liouville problems with eigenparameter dependent boundary conditions
}

\author{
Maozhu Zhang ${ }^{1 *}$, Kun $\mathrm{Li}^{2}$ and Hongxiang Song ${ }^{1}$
}

\section{"Correspondence:}

zhangmaozhu2000@163.com

'College of Mathematics and

Statistics, Taishan University, Taian, China

Full list of author information is

available at the end of the article

\begin{abstract}
In this paper we consider singular Sturm-Liouville problems with eigenparameter dependent boundary conditions and two singular endpoints. The spectrum of such problems can be approximated by those of the inherited restriction operators constructed. Via the abstract operator theory, the strongly resolvent convergence and norm resolvent convergence of a sequence of operators are obtained and it follows that the spectral inclusion of spectrum holds. Moreover, spectral exactness of spectrum holds for two special cases.
\end{abstract}

MSC: 34L15; 34L05

Keywords: Regular approximation; Singular Sturm-Liouville problems; Eigenparameter dependent boundary conditions

\section{Introduction}

We consider the following Sturm-Liouville differential equation:

$$
l y:=\frac{1}{w}\left[-\left(p y^{\prime}\right)^{\prime}+q y\right]=\lambda y, \quad x \in(a, b),
$$

where $\lambda$ is a complex parameter, $-\infty \leq a<b \leq+\infty, \frac{1}{p}, q, w \in L_{\mathrm{loc}}(a, b), p, w>0$ almost everywhere on $(a, b)$.

Assume that $a<a_{0}<b_{0}<b$ and $\lambda_{0} \in \mathbb{R}, u_{1}(x), v_{1}(x)$ are two linearly independent real solutions on the interval $\left(a, a_{0}\right)$ of the equation $l y=\lambda_{0} y$ such that

$$
\left[u_{1}, v_{1}\right](a)=1 .
$$

The eigenparameter dependent boundary condition at $a$ is

$$
\lambda\left(\alpha_{1}\left[y, u_{1}\right](a)+\beta_{1}\left[y, v_{1}\right](a)\right)=\alpha_{2}\left[y, u_{1}\right](a)+\beta_{2}\left[y, v_{1}\right](a)
$$

(c) The Author(s) 2020. This article is licensed under a Creative Commons Attribution 4.0 International License, which permits use, sharing, adaptation, distribution and reproduction in any medium or format, as long as you give appropriate credit to the original author(s) and the source, provide a link to the Creative Commons licence, and indicate if changes were made. The images or other third party material in this article are included in the article's Creative Commons licence, unless indicated otherwise in a credit line to the material. If material is not included in the article's Creative Commons licence and your intended use is not permitted by statutory regulation or exceeds the permitted use, you will need to obtain permission directly from the copyright holder. To view a copy of this licence, visit http://creativecommons.org/licenses/by/4.0/ 
where $\eta=\alpha_{2} \beta_{1}-\alpha_{1} \beta_{2}>0$. If the endpoint $b$ is of limit circle type, then the boundary condition is imposed as

$$
\cos \gamma\left[y, u_{2}\right](b)-\sin \gamma\left[y, v_{2}\right](b)=0,
$$

where $\gamma \in(0, \pi], u_{2}(x), v_{2}(x)$ are two linearly independent real solutions on $\left(b_{0}, b\right)$ of $l y=$ $\tilde{\lambda_{0}} y$ for some $\tilde{\lambda_{0}} \in \mathbb{R}$ such that

$$
\left[u_{2}, v_{2}\right](b)=1 .
$$

If the endpoint $b$ is of limit point type, then there are no boundary conditions imposed.

For a given singular differential operator, its spectrum can be approximated by the eigenvalues of a sequence of regular operators, which plays an important role in the theory of differential boundary value problems and numerical computation of spectrum; see $[2,19,21,25]$. This is an interesting research topic and can be found in $[1,5,7,16,22,24]$. In $[1,5,7,16,23]$, regular approximation of singular differential operator have been studied from second order Sturm-Liouville operators and fourth order operators to even order differential operators and Hamiltonian operators. In [22], Sturm-Liouville problems with transmission conditions are investigated and in [24] Sturm-Liouville problems with eigenparameter dependent boundary conditions and one singular endpoint are considered.

The differential boundary value problems (see [12-14]) with eigenparameter dependent boundary conditions have been widely investigated by many authors such as $[3,4,6,8,9$, $11,17,20,24,26]$ because of the important application in probability theory and physics and so on. In the present paper we consider Sturm-Liouville problems with two singular endpoints and one eigenparameter dependent boundary condition. We find that its spectrum can be approximated by the eigenvalues of a sequence of regular problems, based on the method of the strong graph limit, which is different from that of the previous papers such as $[1,22,24]$. This paper can be regarded as a continuation and extension of [24].

The paper is organized as follows: In Sect. 2, the inherited restriction operators are constructed and are proved to be self-adjoint. In Sect. 3, by the method of strong graph limit, the spectral inclusion of spectrum is obtained. Moreover, in the limit circle case or for the operators bounded below, spectral exactness holds for the eigenvalues below the essential spectrum.

\section{Construction of the induced restriction operators}

Define a Hilbert space

$$
H=L_{w}^{2}(a, b) \oplus \mathbb{C}
$$

with the inner product

$$
(F, G)=(f, g)_{1}+\frac{1}{\eta} f_{1} \overline{g_{1}},
$$

where

$$
F=\left(f, f_{1}\right), G=\left(g, g_{1}\right) \in H, \quad f, g \in L_{w}^{2}(a, b), \quad(f, g)_{1}=\int_{a}^{b} f(x) \overline{g(x)} w(x) d x .
$$


Let

$$
D_{\max }=\left\{y \in L_{w}^{2}(a, b): y, p y^{\prime} \in A C_{\mathrm{loc}}(a, b), l y \in L_{w}^{2}(a, b)\right\} .
$$

Define an operator

$$
A F=\left(l f, \alpha_{2}\left[f, u_{1}\right](a)+\beta_{2}\left[f, v_{1}\right](a)\right)
$$

for any $F \in D(A)$. If the endpoint $b$ is of limit point type, let the domain $D(A)$ of $A$ be

$$
D(A)=\left\{F=\left(f, f_{1}\right) \in H: f \in D_{\max }, f_{1}=\alpha_{1}\left[f, u_{1}\right](a)+\beta_{1}\left[f, v_{1}\right](a)\right\} .
$$

If the endpoint $b$ is of limit circle type, let

$$
\begin{aligned}
D(A)= & \left\{F=\left(f, f_{1}\right) \in H: f \in D_{\max }, f_{1}=\alpha_{1}\left[f, u_{1}\right](a)+\beta_{1}\left[f, v_{1}\right](a),\right. \\
& \left.\cos \gamma\left[f, u_{2}\right](b)-\sin \gamma\left[f, v_{2}\right](b)=0\right\} .
\end{aligned}
$$

Then we have the following lemma.

\section{Lemma 1}

(1) Let $y, z, u, v \in D_{\max }$. If $[v, u]=1$ for some $c, a \leq c \leq b$, then

$$
[y, z](c)=[y, v](c)[\bar{z}, \bar{u}](c)-[y, \bar{u}](c)[\bar{z}, v](c) .
$$

(2) The endpoint $b$ is of the limit point case if and only if $[y, z](b)=0$ for any $y, z \in D_{\max }$.

(3) If the endpoint $a$ is of the limit circle case and $u, v \in D_{\max },[u, v](a)=1$, then there exists a $f \in D_{\max }$ such that $[f, u](a)=\alpha,[f, v](a)=\beta$ for any $\alpha, \beta \in \mathbb{C}$.

Proof See [21], Lemma 10.4.1, 10.4.2, 10.4.6.

In the following we will get the first main result on the operator $A$.

Theorem 1 The operator $A$ defined as above is a densely defined, symmetric and selfadjoint operator in the Hilbert space $H$.

Proof Step 1. We prove the operator $A$ is densely defined. That is to say, for any $F=\left(f, f_{1}\right) \in$ $H$, if $F \perp D(A)$, then $F=0$.

Since $C_{0}^{\infty}(a, b) \oplus\{0\} \subset D(A)$, then, for any $G=(g, 0) \in C_{0}^{\infty}(a, b) \oplus\{0\} \subset D(A)$, we have

$$
(F, G)=\int_{a}^{b} f(x) \overline{g(x)} w(x) d x=0
$$

Therefore $f(x)=0$ almost everywhere on $(a, b)$. On the other hand, for any $G=\left(g, g_{1}\right) \in$ $D(A)$, it follows from $(F, G)=\frac{1}{\eta} f_{1} \overline{g_{1}}=0$ that $f_{1}=0$. 
Step 2. The operator $A$ is symmetric in $H$. For any $F, G \in D(A)$,

$$
\begin{aligned}
(A F, G)-(F, A G)= & \int_{a}^{b} l f(x) \overline{g(x)} w(x) d x+\frac{1}{\eta}\left(\alpha_{2}\left[f, u_{1}\right](a)+\beta_{2}\left[f, v_{1}\right](a)\right) \overline{g_{1}} \\
& -\int_{a}^{b} f(x) \overline{\lg (x)} w(x) d x-\frac{1}{\eta} f_{1} \overline{\alpha_{2}\left[g, u_{1}\right](a)+\beta_{2}\left[g, v_{1}\right](a)} \\
= & \int_{a}^{b}\left[-\left(p f^{\prime}\right)^{\prime}+q f\right] \bar{g} d x-\int_{a}^{b} f\left[-\left(p \bar{g}^{\prime}\right)^{\prime}+q \bar{g}\right] d x \\
& +\frac{1}{\eta}\left(\alpha_{2}\left[f, u_{1}\right](a)+\beta_{2}\left[f, v_{1}\right](a)\right) \overline{\alpha_{1}\left[g, u_{1}\right](a)+\beta_{1}\left[g, v_{1}\right](a)} \\
& -\frac{1}{\eta}\left(\alpha_{1}\left[f, u_{1}\right](a)+\beta_{1}\left[f, v_{1}\right](a)\right) \overline{\alpha_{2}\left[g, u_{1}\right](a)+\beta_{2}\left[g, v_{1}\right](a)} \\
= & {\left.[f, g]\right|_{a} ^{b}-\operatorname{det}\left(\begin{array}{ll}
{\left[f, u_{1}\right](a)} & {\left[f, v_{1}\right](a)} \\
{\left[u_{1}, g\right](a)} & {\left[v_{1}, g\right](a)}
\end{array}\right) . }
\end{aligned}
$$

It follows from Lemma 1(1) that

$$
(A F, G)-(F, A G)=\left.[f, g]\right|_{a} ^{b}+[f, g](a)\left[\overline{u_{1}}, v_{1}\right](a) .
$$

Since $\left[u_{1}, v_{1}\right](a)=1$ and $u_{1}$ is a real function,

$$
(A F, G)-(F, A G)=\left.[f, g]\right|_{a} ^{b}+[f, g](a)=[f, g](b) .
$$

If the endpoint $b$ is of the limit point case, then $[f, g](b)=0$ for any $F, G \in D(A)$ by Lemma $1(2)$. If $b$ is of the limit circle case, then $f, \bar{g}$ satisfy the boundary condition (1.3). It follows from $\left[u_{2}, v_{2}\right](b)=1$ that

$$
\begin{aligned}
{[f, g](b) } & =[f, g](b)\left[u_{2}, v_{2}\right](b)=\operatorname{det}\left(\begin{array}{ll}
{\left[f, u_{2}\right](b)} & {\left[f, v_{2}\right](b)} \\
{\left[\bar{g}, u_{2}\right](b)} & {\left[\bar{g}, v_{2}\right](b)}
\end{array}\right) \\
& =\left[f, u_{2}\right](b)\left[\bar{g}, v_{2}\right](b)-\left[f, v_{2}\right](b)\left[\bar{g}, u_{2}\right](b)=0 .
\end{aligned}
$$

Step 3. The operator $A$ is self-adjoint. For any $F=\left(f, f_{1}\right) \in D(A)$ and some $K \in H$, $(A F, G)=(F, K)$, we prove that $G \in D(A)$ and $A G=K$. It is sufficient to prove that when $b$ is of the limit point case

(1) $g, p g^{\prime} \in A C_{\mathrm{loc}}(a, b), \lg \in L_{w}^{2}(a, b), k=\lg$,

(2) $g_{1}=\alpha_{1}\left[g, u_{1}\right](a)+\beta_{1}\left[g, v_{1}\right](a)$,

(3) $k_{1}=\alpha_{2}\left[g, u_{1}\right](a)+\beta_{2}\left[g, v_{1}\right](a)$,

hold and when $b$ is of the limit circle case, in addition to (1), (2), and (3), the equality

(4) $\cos \gamma\left[g, u_{2}\right](b)-\sin \gamma\left[g, v_{2}\right](b)=0$

holds.

For any $f \in C_{0}^{\infty}(a, b), F=(f, 0) \in D(A)$, then

$$
(A F, G)=\int_{a}^{b} l f \bar{g} w(x) d x=\int_{a}^{b} f(x) \overline{k(x)} w(x) d x=(f, k)_{1}
$$


by $(A F, G)=(F, K)$. It follows from the classical Sturm-Liouville theory that (1) holds. Hence

$$
\begin{aligned}
(A F, G)-(F, K) & \\
= & \int_{a}^{b} l f(x) \overline{g(x)} w(x) d x+\frac{1}{\eta}\left(\alpha_{2}\left[f, u_{1}\right](a)+\beta_{2}\left[f, v_{1}\right](a)\right) \overline{g_{1}} \\
& -\int_{a}^{b} f(x) \overline{k(x)} w(x) d x-\frac{1}{\eta}\left(\alpha_{1}\left[f, u_{1}\right](a)+\beta_{1}\left[f, v_{1}\right](a)\right) \overline{k_{1}} \\
= & \int_{a}^{b} l f(x) \overline{g(x)} w(x) d x+\frac{1}{\eta}\left(\alpha_{2}\left[f, u_{1}\right](a)+\beta_{2}\left[f, v_{1}\right](a)\right) \overline{g_{1}} \\
& -\int_{a}^{b} f(x) \overline{\lg (x)} w(x) d x-\frac{1}{\eta}\left(\alpha_{1}\left[f, u_{1}\right](a)+\beta_{1}\left[f, v_{1}\right](a)\right) \overline{k_{1}} \\
= & {\left.[f, g]\right|_{a} ^{b}+\frac{1}{\eta}\left(\alpha_{2}\left[f, u_{1}\right](a)+\beta_{2}\left[f, v_{1}\right](a)\right) \overline{g_{1}} } \\
& -\frac{1}{\eta}\left(\alpha_{1}\left[f, u_{1}\right](a)+\beta_{1}\left[f, v_{1}\right](a)\right) \overline{k_{1}}=0 .
\end{aligned}
$$

If $b$ is of the limit point case, then $[f, g](b)=0$. Thus

$$
[f, g](a)=\frac{1}{\eta}\left(\alpha_{2}\left[f, u_{1}\right](a)+\beta_{2}\left[f, v_{1}\right](a)\right) \overline{g_{1}}-\frac{1}{\eta}\left(\alpha_{1}\left[f, u_{1}\right](a)+\beta_{1}\left[f, v_{1}\right](a)\right) \overline{k_{1}} \text {. }
$$

It follows from $\left[u_{1}, v_{1}\right](a)=1$ and Lemma $1(1)$ that

$$
[f, g](a)=[f, g](a)\left[u_{1}, v_{1}\right](a)=\left[f, u_{1}\right](a)\left[\bar{g}, v_{1}\right](a)-\left[f, v_{1}\right](a)\left[\bar{g}, u_{1}\right](a) .
$$

It follows from Lemma 1(3) that there exists a function $f \in D_{\max }$ such that $\left[f, u_{1}\right](a)=1$, $\left[f, v_{1}\right](a)=0$. Inserting these into (2.2) and (2.3) we have

$$
\alpha_{2} \overline{g_{1}}-\alpha_{1} \overline{k_{1}}=\eta\left[\bar{g}, v_{1}\right](a)
$$

Similarly we can obtain

$$
\beta_{2} \overline{g_{1}}-\beta_{1} \overline{k_{1}}=-\eta\left[\bar{g}, u_{1}\right](a)
$$

Thus

$$
g_{1}=\alpha_{1}\left[g, u_{1}\right](a)+\beta_{1}\left[g, v_{1}\right](a), \quad k_{1}=\alpha_{2}\left[g, u_{1}\right](a)+\beta_{2}\left[g, v_{1}\right](a) .
$$

Items (2) and (3) follow.

If $b$ is of the limit circle case, then we can choose $F \in D(A)$ such that $f=0$ in the right neighborhood of $a$ and $\cos \gamma\left[f, u_{2}\right](b)-\sin \gamma\left[f, v_{2}\right](b)=0$. Therefore $\left[f, u_{1}\right](a)=\left[f, v_{1}\right](a)=$ $[f, g](a)=0$. Inserting these into $(2.1)$, we can obtain

$$
0=[f, g](b)=[f, g](b)\left[u_{2}, v_{2}\right](b)=\operatorname{det}\left(\begin{array}{ll}
{\left[f, u_{2}\right](b)} & {\left[f, v_{2}\right](b)} \\
{\left[\bar{g}, u_{2}\right](b)} & {\left[\bar{g}, v_{2}\right](b)}
\end{array}\right)
$$


It follows that (4) holds. Choose $f$ such that $f=0$ in the left neighborhood of $b$ and $\left[f, u_{1}\right](a)=0,\left[f, v_{1}\right](a)=1$ or $f=0$ in the left neighborhood of $b$ and $\left[f, u_{1}\right](a)=1$, $\left[f, v_{1}\right](a)=0$, similarly to the proof in the limit point case, we can conclude (2) and (3) hold.

Let

$$
a<a_{r}<a_{0}<b_{0}<b_{r}<b, \quad r \in \mathbb{N}^{+}=\{1,2, \ldots\}
$$

and $\left\{a_{r}\right\}$ is a strictly decreasing sequence with $a_{r} \rightarrow a$ and $\left\{b_{r}\right\}$ is a strictly increasing sequence with $b_{r} \rightarrow b$. Define the Hilbert space

$$
H_{r}=L_{w}^{2}\left(a_{r}, b_{r}\right) \oplus \mathbb{C}
$$

with the inner product

$$
\left(F_{r}, G_{r}\right)=(f, g)_{1 r}+\frac{1}{\eta} f_{1 r} \overline{g_{1 r}}
$$

where

$$
\begin{aligned}
& F_{r}=\left(f, f_{1 r}\right), G_{r}=\left(g, g_{1 r}\right) \in H_{r}, \quad f, g \in L_{w}^{2}\left(a_{r}, b_{r}\right), \quad(f, g)_{1 r}=\int_{a_{r}}^{b_{r}} f(x) \overline{g(x)} w(x) d x, \\
& f_{1 r}=\alpha_{1}\left[f, u_{1}\right]\left(a_{r}\right)+\beta_{1}\left[f, v_{1}\right]\left(a_{r}\right), \quad g_{1 r}=\alpha_{1}\left[g, u_{1}\right]\left(a_{r}\right)+\beta_{1}\left[g, v_{1}\right]\left(a_{r}\right) .
\end{aligned}
$$

Let the maximum domain and the minimum domain as follows:

$$
\begin{aligned}
& D_{\max }^{r}=\left\{y \in L_{w}^{2}\left(a_{r}, b_{r}\right): y, p y^{\prime} \in A C\left(a_{r}, b_{r}\right), l y \in L_{w}^{2}\left(a_{r}, b_{r}\right)\right\}, \\
& D_{\text {min }}^{r}=\left\{y \in D_{\text {max }}^{r}:[y, z]\left(a_{r}\right)-[y, z]\left(b_{r}\right)=0, \text { for all } z \in D_{\text {max }}^{r}\right\} .
\end{aligned}
$$

Define an operator

$$
A_{r} F_{r}=\left(l f, \alpha_{2}\left[f, u_{1}\right]\left(a_{r}\right)+\beta_{2}\left[f, v_{1}\right]\left(a_{r}\right)\right)
$$

for any $F_{r}=\left(f, f_{1 r}\right) \in D\left(A_{r}\right)$. If the endpoint $b$ is of the limit point case, then let the domain of $A_{r}$ be

$$
\begin{aligned}
D\left(A_{r}\right)= & \left\{F_{r}=\left(f, f_{1 r}\right) \in H_{r}: f \in D_{\max }^{r},\right. \\
& \left.f_{1 r}=\alpha_{1}\left[f, u_{1}\right]\left(a_{r}\right)+\beta_{1}\left[f, v_{1}\right]\left(a_{r}\right),\left[f, \varphi_{r}\right]\left(b_{r}\right)=0\right\},
\end{aligned}
$$

where $\varphi_{r} \in D_{\max }^{r}, \varphi_{r} \notin D_{\min }^{r},\left[\varphi_{r}, \varphi_{r}\right]\left(b_{r}\right)=0$. In particular $\varphi_{r}=\left.\varphi\right|_{\left(b_{0}, b_{r}\right)}$ and $\varphi$ can be chosen to be any non-trivial real solution of (1.1) for some $\lambda \in \mathbb{R}$ on $\left(b_{0}, b\right)$. If the endpoint $b$ is of the limit circle case, let

$$
\begin{aligned}
D\left(A_{r}\right)= & \left\{F_{r}=\left(f, f_{1 r}\right) \in H_{r}: f \in D_{\max }^{r}, f_{1 r}=\alpha_{1}\left[f, u_{1}\right]\left(a_{r}\right)+\beta_{1}\left[f, v_{1}\right]\left(a_{r}\right),\right. \\
& \left.\cos \gamma\left[f, u_{2}\right]\left(b_{r}\right)-\sin \gamma\left[f, v_{2}\right]\left(b_{r}\right)=0\right\} .
\end{aligned}
$$


The operator $A_{r}$ is usually called an inherited restriction operator. Then we have the following.

Theorem 2 The operator $A_{r}$ defined as above is a self-adjoint operator in the Hilbert space $H_{r}$ for any $r \in \mathbb{N}^{+}$.

Proof It is obvious that $A_{r}$ is densely defined. In the following we prove $A_{r}$ is symmetric. For any $F_{r}, G_{r} \in D\left(A_{r}\right)$, then

$$
\left(A_{r} F_{r}, G_{r}\right)-\left(F_{r}, A_{r} G_{r}\right)=\left.[f, g]\right|_{a_{r}} ^{b_{r}}+[f, g]\left(a_{r}\right)\left[u_{1}, v_{1}\right]\left(a_{r}\right) .
$$

Since $\left[u_{1}, v_{1}\right]\left(a_{r}\right)=\left[u_{1}, v_{1}\right](a)=1$, thus

$$
\left(A_{r} F_{r}, G_{r}\right)-\left(F_{r}, A_{r} G_{r}\right)=[f, g]\left(b_{r}\right) .
$$

If the endpoint $b$ is of the limit point case, then $[f, \varphi]\left(b_{r}\right)=[g, \varphi]\left(b_{r}\right)=0$. Thus

$$
[f, g]\left(b_{r}\right)=0 .
$$

If $b$ is of the limit circle case, then $f, g$ satisfy

$$
\cos \gamma\left[f, u_{2}\right]\left(b_{r}\right)-\sin \gamma\left[f, v_{2}\right]\left(b_{r}\right)=\cos \gamma\left[g, u_{2}\right]\left(b_{r}\right)-\sin \gamma\left[g, v_{2}\right]\left(b_{r}\right)=0 .
$$

Together with $\left[u_{2}, v_{2}\right]\left(b_{r}\right)=\left[u_{2}, v_{2}\right](b)=1$, we can conclude that

$$
[f, g]\left(b_{r}\right)=[f, g]\left(b_{r}\right)\left[u_{2}, v_{2}\right]\left(b_{r}\right)=\operatorname{det}\left(\begin{array}{ll}
{\left[f, u_{2}\right]\left(b_{r}\right)} & {\left[f, v_{2}\right]\left(b_{r}\right)} \\
{\left[\bar{g}, u_{2}\right]\left(b_{r}\right)} & {\left[\bar{g}, v_{2}\right]\left(b_{r}\right)}
\end{array}\right)=0 .
$$

Similarly to the proof of the self-adjointness of $A$ in Theorem 1, the proof is completed.

We define an operator $A_{r}^{\prime}$ in the space $H$ as follows:

$$
A_{r}^{\prime}=A_{r} \oplus \Theta_{r}=A_{r} P_{r}
$$

with the domain $D\left(A_{r}^{\prime}\right)=D\left(A_{r}\right) \oplus H_{r}^{\perp}$, where $P_{r}$ is the orthogonal projection of $H$ onto $H_{r}$, $\Theta$ is the zero operator in the space $H_{r}^{\perp}=L_{w}^{2}\left(a, a_{r}\right) \oplus L_{w}^{2}\left(b_{r}, b\right)$. It is obvious that $A_{r}^{\prime}$ is also a self-adjoint operator in the space $H$.

\section{Convergence of operators and of spectrum}

In this section we shall give the convergence of the inherited restriction operators and of spectrum. Some fundamental concepts and lemmas are needed, which can be found in $[1,10,15,18]$.

Definition 1 Let $A_{n}, A$ be self-adjoint operators. Then $A_{n}$ is said to converge to $A$ in the strong resolvent sense if $\left(A_{n}-\lambda I\right)^{-1} \rightarrow(A-\lambda I)^{-1}$ strongly for all $\lambda$ with $\Im \lambda \neq 0 . A_{n}$ is said to converge to $A$ in the norm resolvent sense if $\left(A_{n}-\lambda I\right)^{-1} \rightarrow(A-\lambda I)^{-1}$ in norm for all $\lambda$ with $\Im \lambda \neq 0$. 
Definition 2 Let $A_{n}$ be a sequence of operators on a Hilbert space $H$. We say that $\langle\psi, \varphi\rangle \in$ $H \times H$ is in the strong graph limit of $A_{n}$ if we can find $\psi_{n} \in D\left(A_{n}\right)$ so that $\psi_{n} \rightarrow \psi, A_{n} \psi_{n} \rightarrow$ $\varphi$. We denote the set of pairs in the strong graph limit by $\Gamma_{\infty}^{s}$. If $\Gamma_{\infty}^{s}$ is the graph of an operator $A$ we say that $A$ is the strong graph limit of $A_{n}$.

Lemma 2 Suppose that $A_{n}$ and $A$ are self-adjoint operators. Then $A_{n}$ is convergent to $A$ in the strong resolvent sense if and only if $A$ is the strong graph limit of $A_{n}$.

In the following we can obtain the convergence in the strong resolvent sense, using the method of the strong graph limit, which is different from that of the previous papers $[1,5$, $22,24]$.

Theorem 3 Suppose the operators $A, A_{r}, A_{r}^{\prime}$ are defined as above. Then

(1) $A_{r}^{\prime}$ is convergent to $A$ in the strong resolvent sense;

(2) the spectral inclusion holds. That is to say, if $\lambda \in \sigma(A)$, then there exists $\lambda_{r} \in \sigma\left(A_{r}^{\prime}\right)$ so that $\lambda_{r} \rightarrow \lambda$;

(3) let $\left\{E\left(A_{r}, \lambda\right)\right\},\{E(A, \lambda)\}$ with $\lambda$ not an eigenvalue of $A$ be spectral projection of the operators $A_{r}, A$, respectively, and $P_{r}$ be a unit projection from $H$ to $H_{r}$. Then $E\left(A_{r}, \lambda\right) P_{r}$ converges strongly to $E(A, \lambda)$, i.e. for any $f \in H$, then

$$
\left\|E\left(A_{r}, \lambda\right) P_{r} f-E(A, \lambda) f\right\| \rightarrow 0 .
$$

Proof Firstly we prove that, for any

$$
F=\left(f, f_{1}\right)=\left(f, \alpha_{1}\left[f, u_{1}\right](a)+\beta_{1}\left[f, v_{1}\right](a)\right) \in D(A),
$$

there exists $F_{r}=\left(f_{r}, \alpha_{1}\left[f_{r}, u_{1}\right]\left(a_{r}\right)+\beta_{1}\left[f_{r}, v_{1}\right]\left(a_{r}\right)\right) \in D\left(A_{r}^{\prime}\right)$ so that

$$
F_{r} \rightarrow F, \quad A_{r}^{\prime} F_{r} \rightarrow A F
$$

Indeed, we can let $f_{r 1}=\left.f\right|_{x \in\left(a, b_{0}\right)}$ as $x \in\left(a, b_{0}\right)$. It follows from

$$
\left[f_{r 1}, u_{1}\right]\left(a_{r}\right) \rightarrow\left[f, u_{1}\right](a), \quad\left[f_{r 1}, v_{1}\right]\left(a_{r}\right) \rightarrow\left[f, v_{1}\right](a)
$$

that

$$
\begin{aligned}
& \alpha_{1}\left[f_{r 1}, u_{1}\right]\left(a_{r}\right)+\beta_{1}\left[f_{r 1}, v_{1}\right]\left(a_{r}\right) \rightarrow \alpha_{1}\left[f, u_{1}\right](a)+\beta_{1}\left[f, v_{1}\right](a), \\
& \alpha_{2}\left[f_{r 1}, u_{1}\right]\left(a_{r}\right)+\beta_{2}\left[f_{r 1}, v_{1}\right]\left(a_{r}\right) \rightarrow \alpha_{2}\left[f, u_{1}\right](a)+\beta_{2}\left[f, v_{1}\right](a) .
\end{aligned}
$$

If the endpoint $b$ is of the limit point case, we construct two operators $T_{r}, T$ as follows:

$$
\begin{aligned}
& D\left(T_{r}\right)=\left\{y \in L_{w}^{2}\left(b_{0}, b_{r}\right): y, p y^{\prime} \in A C\left(b_{0}, b_{r}\right), l y \in L_{w}^{2}\left(b_{0}, b_{r}\right),\right. \\
& \left.\tilde{f}\left(b_{0}\right)\left(p y^{\prime}\right)\left(b_{0}\right)-\left(p \tilde{f}^{\prime}\right)\left(b_{0}\right) y\left(b_{0}\right)=0,[y, \varphi]\left(b_{r}\right)=0\right\}, \\
& T_{r} y=\left.A y\right|_{\left(b_{0}, b_{r}\right),}
\end{aligned}
$$




$$
\begin{aligned}
& D(T)=\left\{y \in L_{w}^{2}\left(b_{0}, b\right): y, p y^{\prime} \in A C_{\mathrm{loc}}\left(b_{0}, b\right), l y \in L_{w}^{2}\left(b_{0}, b\right),\right. \\
& \left.\quad f\left(b_{0}\right)\left(p y^{\prime}\right)\left(b_{0}\right)-\left(p f^{\prime}\right)\left(b_{0}\right) y\left(b_{0}\right)=0\right\}, \\
& T y=\left.A y\right|_{\left(b_{0}, b\right) .}
\end{aligned}
$$

Define an operator $T_{r}^{\prime}$ in the space $L_{w}^{2}\left(b_{0}, b\right) \oplus \mathbb{C}$ as follows:

$$
T_{r}^{\prime}=T_{r} \oplus \Theta_{r}=T_{r} P_{r}
$$

with the domain $D\left(T_{r}^{\prime}\right)=D\left(T_{r}\right) \oplus L_{w}^{2}\left(b_{r}, b\right)$. Obviously $T_{r}$ and $T$ are both self-adjoint operators and $T_{r}^{\prime}$ is convergent to $T$ in the strong resolvent sense by Theorem 4.1 and Theorem 6.1 of [1]. By Lemma 2, we can find that, for $\left.f\right|_{\left(b_{0}, b\right)} \in D(T)$, there exists a function $f_{r 2} \in D\left(T_{r}^{\prime}\right)$ such that

$$
\begin{aligned}
& \left.f_{r 2} \rightarrow f\right|_{\left(b_{0}, b\right)},\left.\quad T_{r}^{\prime} f_{r 2} \rightarrow T f\right|_{\left(b_{0}, b\right)}, \\
& \left.f\right|_{\left(b_{0}, b\right)}\left(b_{0}\right)=f_{r 2}\left(b_{0}\right), \quad\left(\left.p f^{\prime}\right|_{\left(b_{0}, b\right)}\right)\left(b_{0}\right)=\left(p f_{r 2}^{\prime}\right)\left(b_{0}\right) .
\end{aligned}
$$

Let

$$
f_{r}= \begin{cases}f_{r 1}, & x \in\left(a, b_{0}\right), \\ f_{r 2}, & x \in\left(b_{0}, b\right) .\end{cases}
$$

Then by (3.1)-(3.4), we get $F_{r} \rightarrow F, A_{r}^{\prime} F_{r} \rightarrow A F$. Therefore the operator $A_{r}^{\prime}$ is convergent to $A$ in the strong resolvent sense by Lemma 2. When the endpoint $b$ is of the limit circle case, similar result can be obtained.

Items (2) and (3) follow from Theorem 3.6 in [1] and this completes the proof.

As both endpoints are of the limit circle case, we have the following results.

Theorem 4 Suppose the operators $A_{r}, A$ are defined as above and the endpoint $b$ is of the limit circle case. Then

(7) the operator A has only point spectrum, i.e. the eigenvalues of (1.1)-(1.3);

(2) the operator $A_{r}$ has only point spectrum;

(3) $\left(A_{r}-\lambda I\right)^{-1} P_{r}$ is convergent to $(A-\lambda I)^{-1}$ in norm for any $\lambda$ with $\Im \lambda \neq 0$ and thus the spectral exactness holds, That is to say, if $A_{r}$ is spectral included and if any limit-point of a sequence $\left\{\lambda_{r} \in \sigma\left(A_{r}\right)\right\}$ belongs to $\sigma(A)$;

(4) let $\left\{E\left(A_{r}, \lambda\right)\right\},\{E(A, \lambda)\}$ with $\lambda$ not an eigenvalue of $A$ be spectral projection of the operators $A_{r}, A$, respectively, and $P_{r}$ be a unit projection from $H$ to $H_{r}$. Then $E\left(A_{r}, \lambda\right) P_{r}$ converges to $E(A, \lambda)$ not only strongly but in norm i.e.

$$
\left\|E\left(A_{r}, \lambda\right) P_{r}-E(A, \lambda)\right\| \rightarrow 0 ;
$$

(5) if the operator $A$ is bounded below and denote by $\lambda_{n}\left(A_{r}\right), \lambda_{n}(A)$ the eigenvalues of $A_{r}$, A with $n \in \mathbb{N}=\{0,1,2, \ldots\}$, then $\lambda_{n}\left(A_{r}\right) \rightarrow \lambda_{n}(A)$ as $r \rightarrow \infty$. 
Proof For any $G=\left(g, g_{1}\right) \in H, \lambda \notin \sigma_{p}(A)$, consider the following equation $A F=\lambda F+G$ :

$$
\left\{\begin{array}{l}
\text { lf }=\lambda f+g, \\
\alpha_{2}\left[f, u_{1}\right](a)+\beta_{2}\left[f, v_{1}\right](a)=\lambda\left(\alpha_{1}\left[f, u_{1}\right](a)+\beta_{1}\left[f, v_{1}\right](a)\right)+g_{1}, \\
\cos \gamma\left[f, u_{2}\right](b)-\sin \gamma\left[f, v_{2}\right](b)=0 .
\end{array}\right.
$$

Assume that $\phi(x, \lambda), \psi(x, \lambda)$ are two linearly independent solutions of $l y=\lambda y$ and satisfy

$$
\left\{\begin{array} { l } 
{ \phi ( c , \lambda ) = 1 , } \\
{ ( p \phi ^ { \prime } ) ( c , \lambda ) = 0 , }
\end{array} \quad \left\{\begin{array}{l}
\psi(c, \lambda)=0, \\
\left(p \psi^{\prime}\right)(c, \lambda)=1 .
\end{array}\right.\right.
$$

Using the variable of constant, we can obtain the solution of the equation $l f=\lambda f+g$ is

$$
f(x)=\phi(x, \lambda) \int_{c}^{x} \psi(\xi, \lambda) g(\xi) d \xi-\psi(x, \lambda) \int_{c}^{x} \phi(\xi, \lambda) g(\xi) d \xi+\tilde{c_{1}} \phi(x, \lambda)+\tilde{c_{2}} \psi(x, \lambda) .
$$

Let

$$
\begin{aligned}
& w_{11}=\left(\alpha_{2}-\lambda \alpha_{1}\right)\left[\phi, u_{1}\right](a)+\left(\beta_{2}-\lambda \beta_{1}\right)\left[\phi, v_{1}\right](a), \\
& w_{21}=\cos \gamma\left[\phi, u_{2}\right](b)-\sin \gamma\left[\phi, v_{2}\right](b), \\
& w_{12}=\left(\alpha_{2}-\lambda \alpha_{1}\right)\left[\psi, u_{1}\right](a)+\left(\beta_{2}-\lambda \beta_{1}\right)\left[\psi, v_{1}\right](a), \\
& w_{22}=\cos \gamma\left[\psi, u_{2}\right](b)-\sin \gamma\left[\psi, v_{2}\right](b), \\
& W(\lambda)=\operatorname{det}\left(\begin{array}{ll}
w_{11} & w_{12} \\
w_{21} & w_{22}
\end{array}\right) .
\end{aligned}
$$

Then $W(\lambda)=0$ if and only if $\lambda$ is an eigenvalue of (1.1)-(1.3). Inserting $f(x)$ into the two boundary conditions, we have

$$
\tilde{c_{1}}=\frac{\operatorname{det}\left(c_{i j}\right)_{2 \times 2}}{W(\lambda)}, \quad \tilde{c_{2}}=\frac{\operatorname{det}\left(d_{i j}\right)_{2 \times 2}}{W(\lambda)}
$$

where

$$
\begin{aligned}
c_{11}= & d_{12} \\
= & g_{1}-\left(\alpha_{2}-\lambda \alpha_{1}\right)\left[\phi(x, \lambda) \int_{c}^{x} \psi(\xi, \lambda) g(\xi) d \xi, u_{1}\right](a) \\
& -\left(\beta_{2}-\lambda \beta_{1}\right)\left[\phi(x, \lambda) \int_{c}^{x} \psi(\xi, \lambda) g(\xi) d \xi, v_{1}\right](a) \\
& +\left(\alpha_{2}-\lambda \alpha_{1}\right)\left[\psi(x, \lambda) \int_{c}^{x} \phi(\xi, \lambda) g(\xi) d \xi, u_{1}\right](a) \\
& +\left(\beta_{2}-\lambda \beta_{1}\right)\left[\psi(x, \lambda) \int_{c}^{x} \phi(\xi, \lambda) g(\xi) d \xi, v_{1}\right](a),
\end{aligned}
$$




$$
\begin{aligned}
c_{21}= & d_{22} \\
= & -\cos \gamma\left[\phi(x, \lambda) \int_{c}^{x} \psi(\xi, \lambda) g(\xi) d \xi, u_{2}\right](b) \\
& +\sin \gamma\left[\phi(x, \lambda) \int_{c}^{x} \psi(\xi, \lambda) g(\xi) d \xi, v_{2}\right](b) \\
& +\cos \gamma\left[\psi(x, \lambda) \int_{c}^{x} \phi(\xi, \lambda) g(\xi) d \xi, u_{2}\right](b) \\
& -\sin \gamma\left[\psi(x, \lambda) \int_{c}^{x} \phi(\xi, \lambda) g(\xi) d \xi, v_{2}\right](b), \\
c_{12}= & w_{12}, \quad c_{22}=w_{22}, \quad d_{11}=w_{11}, \quad d_{21}=w_{21} .
\end{aligned}
$$

Thus the equation $A F=\lambda F+G$ has a unique solution $F \in D(A)$ and the operator $A$ has only point spectrum.

For any $G=\left(g, g_{1}\right) \in H_{r}, \lambda \notin \sigma_{p}\left(A_{r}\right)$, consider the following equation $A_{r} F=\lambda F+G$ :

$$
\left\{\begin{array}{l}
l f=\lambda f+g, \\
\alpha_{2}\left[f, u_{1}\right]\left(a_{r}\right)+\beta_{2}\left[f, v_{1}\right]\left(a_{r}\right)=\lambda\left(\alpha_{1}\left[f, u_{1}\right]\left(a_{r}\right)+\beta_{1}\left[f, v_{1}\right]\left(a_{r}\right)\right)+g_{1}, \\
\cos \gamma\left[f, u_{2}\right]\left(b_{r}\right)-\sin \gamma\left[f, v_{2}\right]\left(b_{r}\right)=0 .
\end{array}\right.
$$

Using the variable of constant, we can obtain the solution of $l f=\lambda f+g$ :

$$
f(x)=\phi(x, \lambda) \int_{c}^{x} \psi(\xi, \lambda) g(\xi) d \xi-\psi(x, \lambda) \int_{c}^{x} \phi(\xi, \lambda) g(\xi) d \xi+{\tilde{c_{1}}}^{r} \phi(x, \lambda)+{\tilde{c_{2}}}^{r} \psi(x, \lambda)
$$

Similarly to the above proof, $A_{r}$ has only a point spectrum and $\tilde{c_{1}}{ }^{r} \rightarrow \tilde{c_{1}}, \tilde{c_{2}}{ }^{r} \rightarrow \tilde{c_{2}}$. Items (3), (4) and (5) follow.

As the endpoint $a$ is of the limit circle case and $b$ is of the limit point case, we can construct special inherited restriction operators such that spectral exactness holds.

Theorem 5 Suppose the operator $A$ is defined as above and $\left\{E\left(A_{r}, \lambda\right)\right\},\{E(A, \lambda)\}, P_{r}$ are defined as in Theorem 4,

(1) The endpoint $b$ is of the limit point case.

(2) The operator $A$ is bounded below. If there exist finite eigenvalues below the essential spectrum $\sigma_{e}(A)$, let these eigenvalues be $\lambda_{n}(A)$ with $n=0,1,2, \ldots, n_{0}$. If there exist infinite eigenvalues below $\sigma_{e}(A)$, denote by $\lambda_{n}(A)$ with $n \in \mathbb{N}$ all the eigenvalues.

(3) The operator $\tilde{A}_{r}$ is defined as follows:

$$
\begin{aligned}
& D\left(\tilde{A}_{r}\right)=\left\{F_{r}=\left(f, f_{1 r}\right) \in H_{r}: f, p f^{\prime} \in A C\left(a_{r}, b_{r}\right), l f \in L_{w}^{2}\left(a_{r}, b_{r}\right),\right. \\
&\left.f_{1 r}=\alpha_{1}\left[f, u_{1}\right]\left(a_{r}\right)+\beta_{1}\left[f, v_{1}\right]\left(a_{r}\right), f\left(b_{r}\right)=0\right\}, \\
& \tilde{A}_{r} F_{r}=A_{r} F_{r} ;
\end{aligned}
$$

let $\left\{E\left(\tilde{A}_{r}, \lambda\right)\right\},\{E(A, \lambda)\}$ with $\lambda$ not an eigenvalue of $A$ be spectral projection of the operators $\tilde{A}_{r}, A$, respectively, and $P_{r}$ be a unit projection from $H$ to $H_{r}$. 
Then

(1) $E\left(\tilde{A}_{r}, \lambda\right) P_{r}$ converges to $E(A, \lambda)$ not only strongly but in norm.

(2) Spectral exactness holds and hence $\lambda_{n}\left(\tilde{A}_{r}\right) \rightarrow \lambda_{n}(A), r \rightarrow \infty$.

Proof Define a Hilbert space $\hat{H}_{r}=L_{w}^{2}\left(a_{r}, b\right)$ and an operator $\hat{A}_{r}$ as follows:

$$
\begin{aligned}
& D\left(\hat{A_{r}}\right)=\left\{Y_{r}=\left(y_{r}, y_{1 r}\right) \in \hat{H}_{r} \oplus C, y_{r}, p y_{r}^{\prime} \in A C_{\mathrm{loc}}\left(a_{r}, b\right),\right. \\
& \left.\quad l y_{r} \in L_{w}^{2}\left(a_{r}, b\right), y_{1 r}=\alpha_{1}\left[y_{r}, u_{1}\right]\left(a_{r}\right)+\beta_{1}\left[y_{r}, v_{1}\right]\left(a_{r}\right)\right\} ; \\
& \hat{A}_{r} Y_{r}=\left(l y_{r}, \alpha_{2}\left[y_{r}, u_{1}\right]\left(a_{r}\right)+\beta_{2}\left[y_{r}, v_{1}\right]\left(a_{r}\right)\right) .
\end{aligned}
$$

Let $\lambda \in \mathbb{C} \backslash \mathbb{R}$. Let $u_{l}$ be a solution of $(1.1)$ on $(a, b)$ which satisfies the boundary condition (1.2). It is obvious that $u_{l}$ is unique up to a constant. Let $u_{r}$ be a solution of (1.1) lying in $L_{w}^{2}(a, b)$. Since $b$ is of the limit point case, then $u_{r}$ is also unique up to a constant. It follows that $u_{l}, u_{r}$ are two linearly independent functions and then we can choose suitable constants such that $W\left[u_{l}, u_{r}\right]=1$. Denote by $R(s, t, \lambda), R_{r}(s, t, \lambda)$ the kernels of $(A-\lambda I)^{-1}$, $\left(\hat{A}_{r}-\lambda I\right)^{-1}$, then [18]

$$
R(s, t, \lambda)-R_{r}(s, t, \lambda)= \begin{cases}u_{l}(s) u_{r}(t), & a<s<a_{r}, s \leq t<b, \\ -c_{r} u_{r}(s) u_{r}(t), & a_{r} \leq s<t<b, a_{r} \leq t<s<b, \\ u_{r}(s) u_{l}(t), & a<t<a_{r}, t<s<b .\end{cases}
$$

By Theorem 3, $\left(\hat{A}_{r}-\lambda I\right)^{-1} P_{r}$ is strongly convergent to $(A-\lambda I)^{-1}$. Hence $c_{r} \rightarrow 0$ as $r \rightarrow \infty$. Thus $\left(\hat{A}_{r}-\lambda I\right)^{-1} P_{r}$ is convergent to $(A-\lambda I)^{-1}$ in norm and $\hat{A}_{r}$ is spectral exact for $A$. Therefore as $r$ is sufficiently large, for any $\lambda$ below $\sigma_{e}(A)$, we have

$$
\operatorname{dim} E\left(\hat{A}_{r}, \lambda\right)=\operatorname{dim} E(A, \lambda)<+\infty
$$

On the other hand, the closed form of $\tilde{A}_{r}$ is a restriction of $\hat{A}_{r}$ on $\left(a_{r}, b_{r}\right)$ and thus

$$
\operatorname{dim} E\left(\tilde{A}_{r}, \lambda\right) \leq \operatorname{dim} E\left(\hat{A}_{r}, \lambda\right)=\operatorname{dim} E(A, \lambda)<+\infty
$$

By Theorem $3, E\left(\tilde{A}_{r}, \lambda\right) P_{r}$ is strongly convergent to $E(A, \lambda)$. Hence $E\left(\tilde{A}_{r}, \lambda\right) P_{r}$ is convergent to $E(A, \lambda)$ in norm by Lemmas 1.23 and 1.24 in Chap. 8 of [10]. Item (2) follows.

Acknowledgements

The authors thank the referees for his/her comments and detailed suggestions. These have significantly improved the presentation of this paper.

Funding

This research was partially supported by the Natural Science Foundation of Shandong Province (ZR2017MA042,

ZR2018MF019) and China Postdoctoral Science Foundation (2019M662313).

Availability of data and materials

Not applicable.

Competing interests

The authors declare that there is no competing interests. 


\section{Author details}

${ }^{1}$ College of Mathematics and Statistics, Taishan University, Taian, China. ${ }^{2}$ School of Mathematics Sciences, Qufu Normal University, Qufu, China.

\section{Publisher's Note}

Springer Nature remains neutral with regard to jurisdictional claims in published maps and institutional affiliations.

Received: 6 November 2019 Accepted: 17 December 2019 Published online: 06 January 2020

\section{References}

1. Bailey, P.B., Everitt, W.N., Weidmann, J., Zettl, A.: Regular approximations of singular Sturm-Liouville problems. Results Math. 23, 3-22 (1993)

2. Bailey, P.B., Everitt, W.N., Zettl, A.: The SLEIGN2 Sturm-Liouville code, ACM TOMS. ACM Trans. Math. Softw. 21, 1-15 (2001)

3. Binding, P.A., Browne, P.J., Seddighi, K.: Sturm-Liouville problems with eigenparameter dependent boundary conditions. Proc. Edinb. Math. Soc. 37(2), 57-72 (1993)

4. Binding, P.A., Browne, P.J., Watson, B.A.: Equivalence of inverse Sturm-Liouville problems with boundary conditions rationally dependent on the eigenparameter. J. Math. Anal. Appl. 291, 246-261 (2004)

5. Brown, M., Greenberg, L., Marletta, M.: Convergence of regular approximations to the spectra of singular fourth order Sturm-Liouville problems. Proc. R. Soc. Edinb., Sect. A 128(5), 907-944 (1998)

6. Cai, J., Zheng, Z:: Matrix representations of Sturm-Liouville problems with coupled eigenparameter-dependent boundary conditions and transmission conditions. Math. Methods Appl. Sci. 41, 3495-3508 (2018)

7. El-Gebeily, M.A.: Regular approximation of singular self-adjoint differential operators. IMA J. Appl. Math. 68, 471-489 (2003)

8. Fulton, C.T.: Two-point boundary value problems with eigenvalue parameter contained in the boundary conditions. Proc. R. Soc. Edinb. A 77, 293-308 (1977)

9. Fulton, C.T.: Singular eigenvalue problems with eigenvalue parameter contained in the boundary conditions. Proc. R. Soc. Edinb. A 87, 1-34 (1980)

10. Kato, T.: Pertubation Theory for Linear Operators, 2nd edn. Springer, Heidelberg (1980)

11. Mukhtarov, O.S., Aydemir, K.: Eigenfunction expansion for Sturm-Liouville problems with transmission conditions at one interior point. Acta Math. Sci. Ser. B Engl. Ed. 35(3), 639-649 (2015)

12. Nursultanov, M., Rozenblum, G.: Eigenvalue asymptotics for the Sturm-Liouville operator with potential having a strong local negative singularity. Opusc. Math. 37(1), 109-139 (2017)

13. Papageorgiou, N., Radulescu, V., Repovs, D.: Nonlinear Analysis-Theory and Methods. Springer Monographs in Mathematics. Springer, Cham (2019)

14. Radulescu, V.: Finitely many solutions for a class of boundary value problems with superlinear convex nonlinearity. Arch. Math. (Basel) 84(6), 538-550 (2005)

15. Reed, M., Simon, B.: Methods of Modern Mathematical Physics I: Functional Analysis. Academic Press, San Diego (1972)

16. Teschl, G.: On the approximation of isolated eigenvalues of ordinary differential operators. Proc. Am. Math. Soc. 136(7), 2473-2476 (2008)

17. Walter, J.: Regular eigenvalue problems with eigenvalue parameter in the boundary condition. Math. Z. 133, 301-312 (1973)

18. Weidmann, J.: Linear Operators in Hilbert Space. Springer, New York (1980)

19. Weidmann, J.: Spectral Theory of Ordinary Differential Operators. Lectures Notes in Math., vol. 1258. Springer, Berlin (1987)

20. Yang, C., Bondarenko, N., Xu, X.: An inverse problem for the Sturm-Liouville pencil with arbitrary entire functions in the boundary condition. Inverse Probl. Imaging 14(1), 153-169 (2020)

21. Zettl, A.: Sturm-Liouville Theory. Mathematical Surveys Monographs, vol. 121. Am. Math. Soc., Providence (2005)

22. Zhang, M.: Regular approximation of singular Sturm-Liouville problems with transmission conditions. Appl. Math. Comput. 247, 511-520 (2014)

23. Zhang, M., Li, K., Wang, Y.: Regular approximation of linear Hamiltonian operators with two singular endpoints. J. Math. Anal. Appl. (2020). https://doi.org/10.1016/j.jmaa.2019.123758

24. Zhang, M., Sun, J., Zettl, A.: The spectrum of singular Sturm-Liouville problems with eigenparameter dependent boundary conditions and its approximation. Results Math. 63, 1311-1330 (2013)

25. Zhang, M., Sun, J., Zettl, A.: Eigenvalues of limit-point Sturm-Liouville problems. J. Math. Anal. Appl. 419, 627-642 (2014)

26. Zheng, Z., Cai, J., Li, K., Zhang, M.: A discontinuous Sturm-Liouville problem with boundary conditions rationally dependent on the eigenparameter. Bound. Value Probl. 2018, 103 (2018). https://doi.org/10.1186/s13661-018-1023-x 